\title{
INVESTIGACIÓN DE MATERIAS PRIMAS PARA LA INDUSTRIA ALAFARERA EN EL CORREDOR PALORA - SAN JUAN BOSCO DE LA PROVINCIA DE MORONA SANTIAGO
}

\author{
RAW MATERIALS RESEARCH FOR THE POTTERY INDUSTRY IN THE CORRIDOR \\ PALORA - SAN JUAN BOSCO FROM THE MORONA SANTIAGO PROVINCE

\section{EDGAR RAMIRO PINO LOZA; MARCO ANTONIO MEJÍA FLORES; JAZMÍN GABRIELA JARAMILLO ALTAMIRANO}

FACULTAD DE RECURSOS NATURALES, ESCUELA SUPERIOR POLITÉCNICA DEL CHIMBORAZO EXT. MORONA SANTIAGO - ESPOCH. Don Bosco y José Félix Pintado, s/n. Macas - Ecuador edgar.pino@espoch.edu.ec; marco.mejia@espoch.edu.ec; gabby_j93@ hotmail.com

Recibido: 27 de octubre 2018 / Aceptado: 30 de mayo de 2019

\author{
RESUMEN
}

En el cantón de Limón Indanza se desarrolla por más de 20 años la actividad extractiva de materiales no metálicos, como: arena silícea, feldespato y caolín; explotados principalmente por las compañías EXPLOSUR y GRAIMAN quienes han efectuado permanentemente trabajos de investigación en la zona, cuya información es de uso exclusivo en las empresas; de igual manera el material es únicamente extraído y transportado en calidad de materia prima a las fábricas en la ciudad de Cuenca. No existe industria local de alfarería o emprendimiento para el aprovechamiento de este recurso y la creación de una nueva alternativa económica es inexistente. La investigación procuro determinar mediante prospección el potencial de materiales cerámicos para uso en alfarería artesanal y pequeña industria en la provincia de Morona Santiago, como también la caracterización de las materias primas cerámicas y su relación con su entorno geológico. La investigación inició con la recopilación de información documental de antecedentes sobre yacimientos no metálicos en Morona Santiago, luego se planifico y ejecuto la prospección en la provincia; se inicia con la recolección de muestras para el posterior análisis de laboratorio, y determinar sus propiedades físico-cerámicas como: su color, plasticidad, refractariedad, porosidad, absorción de agua, contracción a la quema y densidad; lo cual permitió formular una caracterización de cada una de las muestras. Dando como resultado que en la provincia de Morona Santiago existen yacimientos no metálicos aprovechables con buenas características para el uso de cerámica de quema blanca o roja, también se pudo establecer con los resultados de los análisis a las muestras que en el cantón Morona se encuentran los lugares más prospectivos de arcilla para uso cerámico.

Palabras clave: Materia prima - Cerámica - Propiedades físico cerámicas 


\begin{abstract}
For more than twenty years, people in Limon Indanza Canton have developed the extractive activity of nometallic materials such as silica sand, feldspar and kaolin. "EXPLOSUR" and "GRAIMAN" companies are the principal beneficiaries from these materials extraction. These companies develop research works all the time, and the data are confidential and of exclusive use of them. In the same way, EXPLOSUR and GRAIMAN extract and transport the materials as feedstock to their factories in Cuenca city. In Limon Indanza there is not pottery to leverage these resources, therefore the establishment of a new economic alternative is unavailable. The research intended to determine through prospection the potential of ceramic materials for use in artisanal pottery and small industry in the province of Morona Santiago. On the other hand, this research also analyzed the characterization of ceramic feedstock and the relationship within its geological environment. The research began with the compilation of information about documentary evidence of no-metallic deposits in Morona Santiago. After that, it was planned and executed the prospection in the province. It began with the collection of soil samples to be analyzed in a laboratory and determining their physical-ceramic properties such as color, plasticity, refractoriness, porosity, water absorption, shrinkage to burning and density. The results of this process allowed formulating a characterization of each one of the soil samples. The analyzes concluded that in Morona Santiago province there are non-metallic deposits with good characteristics for the use of white and red burning ceramics. It was also possible to establish that in Morona canton there prospective clay sites for ceramic uses.
\end{abstract}

Keywords: feedstock - ceramic - ceramic physical properties

\title{
1. INTRODUCCIÓN
}

Antes de profundizar en la producción cerámica, hay que entender conceptualmente a qué nos referimos. Al decir producción cerámica entendemos una... "serie de tareas encaminadas a la obtención de objetos cerámicos, es decir, una actividad”. ( Daly, 1998).

En Ecuador, las arcillas se presentan prácticamente en todas las formaciones sedimentarias tanto continentales como marinas, sin existir estudios de su composición mineralógica, calidad y reservas, importantes para obtener productos con niveles de calidad altos, en caso de explotación artesanal, mientras en casos específicos de empresas o compañías, han sido estudiadas debidamente los depósitos de acuerdo a sus intereses industriales.(Plan Nacional de Desarrollo del Sector Minero, 2016).

El cantón Limón Indanza de la provincia de Morona Santiago ha proporcionado en los últimos 40 años aproximadamente el $60 \%$ de la materia prima para la industria cerámica, como son: EDESA, FRANZ VIEGENER en Quito; RIALTO, ITALPISOS, GRAIMAN y CERÁMICA ANDINA en Cuenca y ECUATORIANA DE CERÁMICA en Riobamba, además de otras pequeñas empresas cerámicas en todo el país. Y además abastece del $100 \%$ de arena silícea para la producción de envases de vidrio.

El conocimiento del potencial minero en no metálicos está focalizado en el cantón de Limón Indaza y no está difundido a la población, por lo que pasa desapercibido dentro de la planificación estratégica provincial. Esta investigación tiene como propósito conocer las características y el comportamiento que tienen las arcillas y feldespato como materia prima cerámica, y esta información ponerla a disposición de la ciudadanía, de esta manera aportar con una nueva alternativa de producción en la provincia. 


\section{MARCO TEÓRICO}

\subsection{ORIGEN DE LAS ARCILLAS}

Las arcillas son formadas por alteración de las rocas volcánicas y piroclásticos ácidas; este tipo de arcillas, formado principalmente por los minerales del grupo de la caolinita, aparecen en el sur del país y se formó por la alteración de las rocas de la formación Saraguro y la formación Tarqui." (Cargua y Encalada, 1997); en la provincia de Morona Santiago, aparecen estas rocas como productos de alteración de rocas graníticas y de las andesitas Misahualli.

\subsection{ANÁLISIS FÍSICO}

\section{Granulometría}

Análisis granulométrico es el procedimiento mecánico utilizando una tamizadora por un tiempo de 5 minutos por cada muestra con el propósito de separar las partículas constitutivas del agregado según tamaños, de tal manera que se puedan conocer las cantidades en peso de cada tamaño que aporta al peso total. Para separar por tamaños se utilizan las mallas de las aberturas $\mathrm{n}^{\circ} .12,16,20,30,40,50,60,80,100$ y 200 , las cuales proporcionan el tamaño máximo de agregado en cada una de ellas. En la práctica los pesos de cada tamaño se expresan como porcentajes retenidos en cada malla con respecto al total de la muestra. Estos porcentajes retenidos en cada malla se calculan tanto parciales como acumulados, y con estos últimos se procede a trazar la gráfica de valores de material (granulometría).

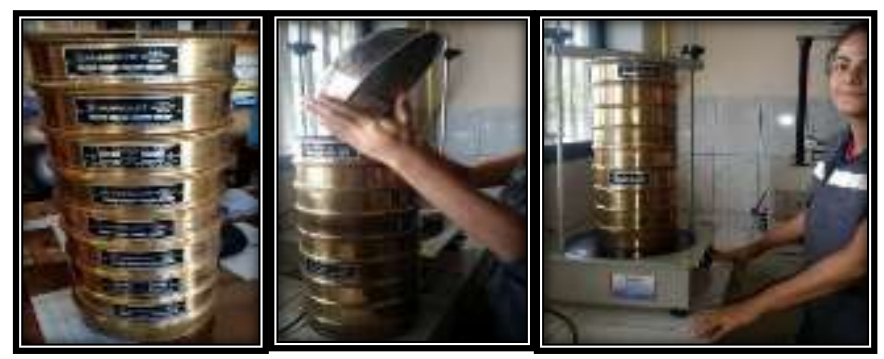

Fotos 1- 3: Ensayo Granulométrico

\section{Análisis de Plasticidad}

La técnica experimental utilizada es de casa grande usada para el estudio del comportamiento de las mezclas de cualquier material de granulometría fina con agua.

Límite líquido.- la determinación del límite líquido se realiza por medio de un ensayo que se encuentra normalizado (norma une 103-103-94). Se comienza amasando con agua destilada, una determinada cantidad de arcilla ( 150 a 200 gramos) que pase por el tamiz de abertura $400 \mu \mathrm{m}$, procurando añadir la cantidad de agua necesaria para acercarse lo más posible al límite líquido, el material retenido por el tamiz de $400 \mu \mathrm{m}$ solo debe consistir en granos de arena, etc. individuales. La masa así obtenida se coloca en una espátula y se pasa a la cuchara de casagrande colocando la masa en la cuchara, se abre un surco o canal con un acanalador normalizado. A continuación se comienza a dar 
vueltas a la manivela, con lo cual, se levanta la cuchara y se deja caer desde la altura de un centímetro y se dan dos golpes por segundo.

Se continúa la operación hasta que las paredes del surco se unan por su fondo en una longitud de $13 \mathrm{~mm}$. Si esto ocurre después de dar exactamente 25 vueltas a la manivela, la arcilla tiene un contenido de humedad correspondiente al límite líquido, sin embargo no será normal que la humedad corresponda a la del límite líquido. Se hacen dos ensayos y se determinan sus correspondientes humedades en tantos por ciento, tomando la arcilla próxima a las paredes del surco, en la parte donde se cerró, para que el ensayo sea válido el número de golpes debe estar comprendido entre 15 y 35 , se ha de obtener una determinación entre 15 y 25 golpes y otra entre 25 y 35, si después de varias determinaciones, el número de golpes requerido para cerrar el surco fuese siempre inferior a 25 significa que no se puede determinar el límite líquido y se debe anotar dicha arcilla como no plástica.

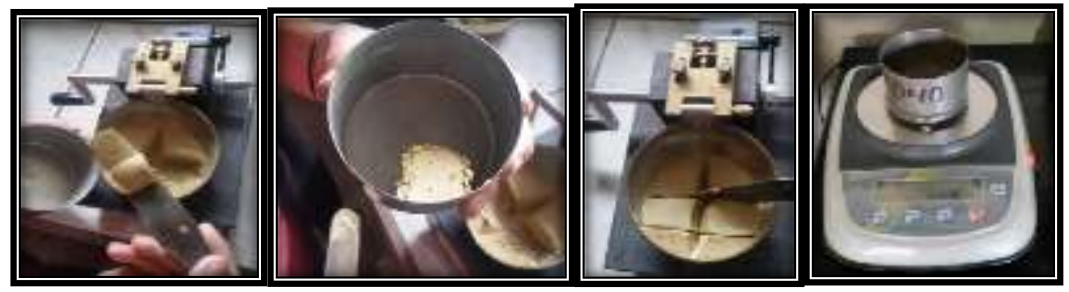

Fotos 4 - 8: Ensayo del Limite Liquido

\section{Límite Plástico}

Según la Norma INEN 685. Mecánica de suelos terminología. Se define al Límite Plástico como el contenido de agua de un suelo remoldeado, en el punto de transición entre los estados plástico y sólido.

El ensayo se realiza con la fracción de arcilla que pasa por el tamiz de abertura $400 \mu \mathrm{m}$, con un contenido de humedad algo superior al límite plástico, con esta humedad será posible formar fácilmente una bola con la arcilla sin que se resquebraje, a continuación se toman unos 8 gramos de dicha arcilla, se forman con ella una especie de elipsoide, y se rueda entre la palma de la mano y una superficie lisa que no absorba mucha humedad, hasta llegar a un diámetro de $3 \mathrm{~mm}$., si al llegar a éste diámetro no ha cuarteado el cilindro de modo que quede dividido en trozos de unos $6 \mathrm{~mm}$ de longitud como media, se vuelve a formar el elipsoide con menor cantidad de humedad y a rodar hasta llegar a dicho tipo de resquebrajamiento, la arcilla se encontrará en su límite plástico cuando se cuartee a los $3 \mathrm{~mm}$ de diámetro.

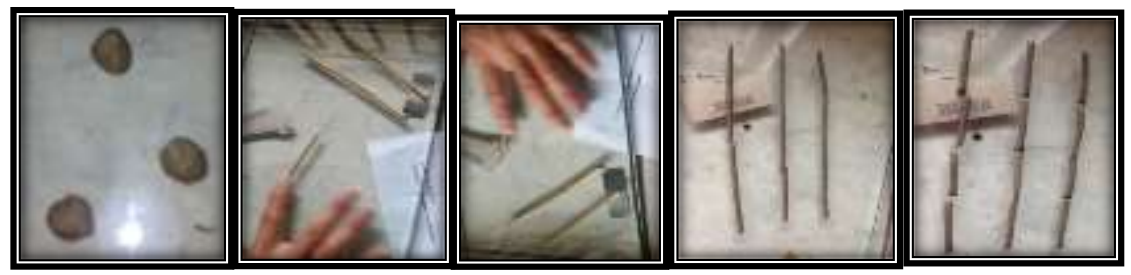

Fotos 9 -13: Ensayo del Limite Plástico 


\subsection{ANÁLISIS TÉCNICO-CERÁMICO}

\section{Elaboración de placas para el análisis técnico-cerámico.}

Se toma la muestra molida, a) se la tamiza con la ayuda del colador metálico, b) el material que no pasa por el colador se queda retenido, pasa solo lo fino para poder hacer la pasta, c) se vierte un poco de agua, d) se amasa la muestra, e) la pasta se la coloca el molde de yeso para eliminar el exceso de agua para que quede más homogénea y trabajable, f) se forma una bola, g) y h) se la expande con la ayuda del tubo y los palos para poder tener el grosor deseado, i) Tomar las medidas con la regla y empezar a cortar con el estilete con mucho cuidado, j ) Ajuste de la placa, k),1) y m) se hizo una raya aguda exactamente de $10 \mathrm{~cm}$ de largo con la ayuda del pie de rey para medir con exactitud. Esta raya se hace con el objetivo de medir la contracción durante el secado en forma natural o al ambiente y después de su cocción. n) y o) en la cara de cada placa se las codifico, p) por último se colocó un pedazo de vidrio para evitar cualquier deformación de la placa.

Se elaboró 3 placas para cada una de las 26 muestras, para luego ser sometidos a la cocción a 3 temperaturas diferentes.

\section{Cocción}

"La cocción es el proceso de transformación de los minerales de arcilla en cerámica" (Rodríguez, y Torrecillas, 2002). "En la cocción de materiales cerámicos no solo es necesario e importante llegar a la temperatura elevada deseada sino las velocidades de calentamiento y de enfriamiento que define el ciclo de quema" (Cargua y Encalada, 1997).

La causa principal de rotura en la cocción de una pieza cruda es la humedad. Cualquier humedad que quede en el barro se transformará en vapor cuando suba la temperatura del horno por encima del punto de ebullición, y se extenderá rompiendo la pieza. "El color (suave cuando la pieza se seca) y el tacto (frio al tocarla cuando todavía está húmeda) son indicios de que la pieza se está secando incluso cuando todos los objetos colocados en un horno para biscocho parecen absolutamente secos, es importante empezar el proceso de calentamiento muy lentamente y abrir las válvulas para dejar escapar el vapor" (Dolors, 2005). Es decir que antes de llevarlas las placas al horno se tuvieron que secar al ambiente por 3 semanas.

Para la cocción de estos materiales cerámicos se utilizó un horno eléctrico del ceramista Iván Encalada el cual se encuentra en el taller cerámico en ciudad de Cuenca. La temperatura que se aplicó para este ensayo fue propuesta especialmente por un ceramista con experiencia, la cual recomendó realizarlas a tres diferentes temperaturas que son a: $1000^{\circ} \mathrm{C}, 1050{ }^{\circ} \mathrm{C}$ y $1200{ }^{\circ} \mathrm{C}$.

La frecuencia correcta de cocción de placas fue la siguiente:

Se colocó las placas en el horno eléctrico. Se programó con un pequeño ordenador construido en el circuito para seguir estas curvas de calor para la primera quema a 1000 ${ }^{\circ} \mathrm{C}$. El horno comenzó a calentarse a baja temperatura de dos a tres horas, con la tapa 
abierta para que pueda salir vapor de agua, para prevenir si es que aún no se han secado en su totalidad las placas, ya que si están húmedas tienen a trisarse o a explotarse, cuando el vapor de agua cesa ya se cierra la tapa del horno para que continúe la cocción de las placas hasta alcanzar la temperatura ya mencionada por 4 horas. Luego de este lapso se apaga el horno y se deja que enfrié a temperatura ambiente. El mismo procedimiento se lo realizo con las temperaturas de $1050{ }^{\circ} \mathrm{C}$ y $1200{ }^{\circ} \mathrm{C}$.
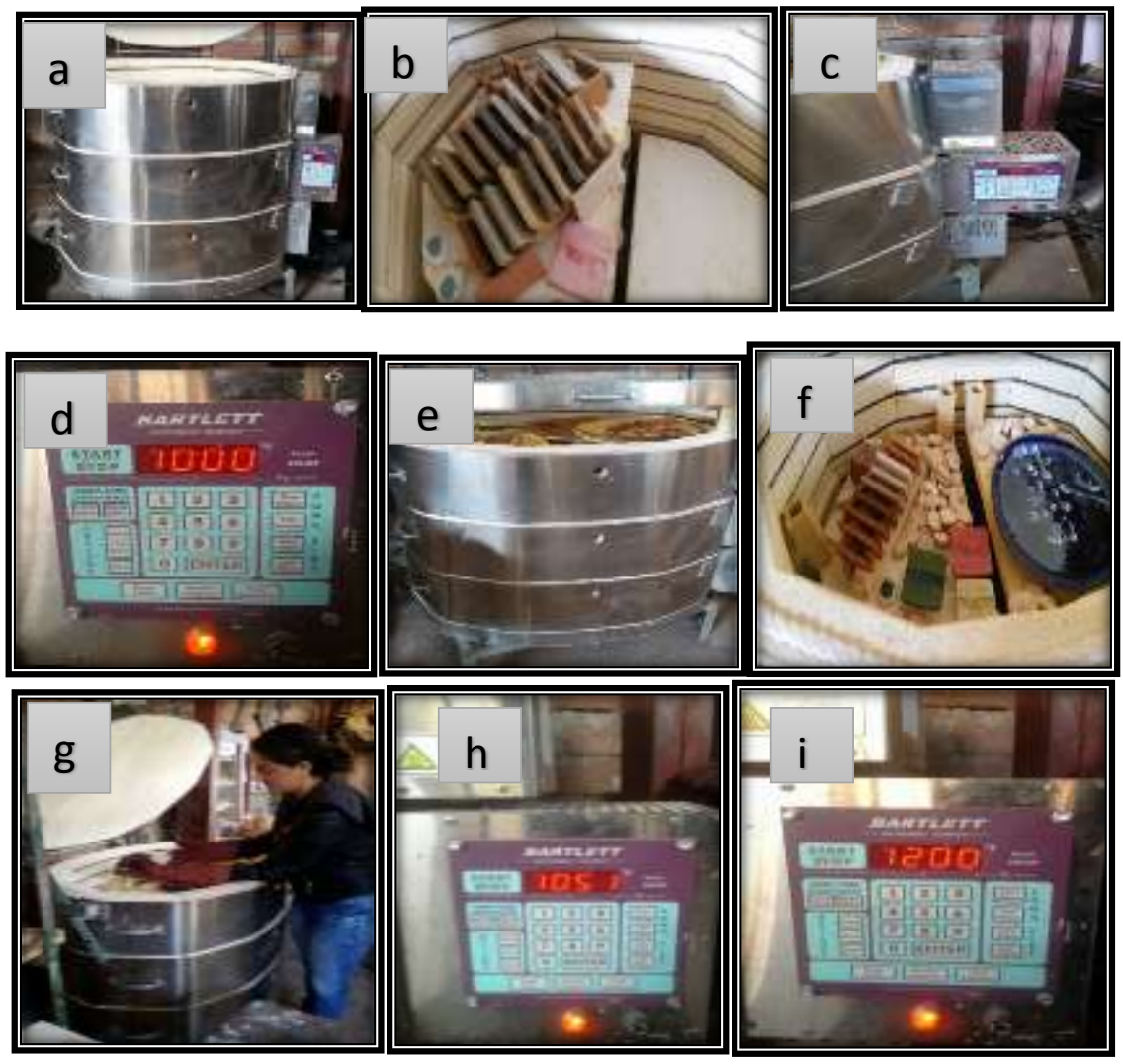

Fotos 14 -22: Cocción. La frecuencia correcta de cocción de placas fue la siguiente a) Horno eléctrico, b) colocación de las placas en el horno, c) la tapa abierta de horno para que pueda salir vapor de agua, d) Temperatura de la primera cocción que se va a realizar a las placas, e) y f) enfriamiento del horno) retirada de las piezas ya cocidas, h) e i) temperaturas de las posteriores cocciones.

\section{Color tras la Cocción.}

"El color de cualquier pasta cerámica dependerá de las condiciones de cocción, el contenido en hierro de la arcilla y la forma en que éste distribuido dentro de la arcilla" (Rhodes, 1990).

Muchas arcillas presentan coloraciones diversas después de la cocción (rosa, rojiza, amarillenta, etc.) debido a la presencia en ella de óxido de hierro. "Los caolines y las arcillas ricas en carbonato cálcico presentan un color blanco después de la cocción" (Caruso, 1986). "El color de la materia prima tras la cocción tiene importancia o deja de tenerla según el uso que se dé a la materia prima" (Cargua y Encalada, 1997). 
En nuestro estudio el color que presentaron las placas tras la cocción fueron la mayor parte optimas para el uso en cerámica roja que se utilizaría para la industria de la construcción, como baldosas, tejas, ladrillos, productos de alfarería, etc. mientras que la menor parte fue para cerámica blanca, que se usa para porcelana blanca, cerámica fina y cerámica sanitaria.

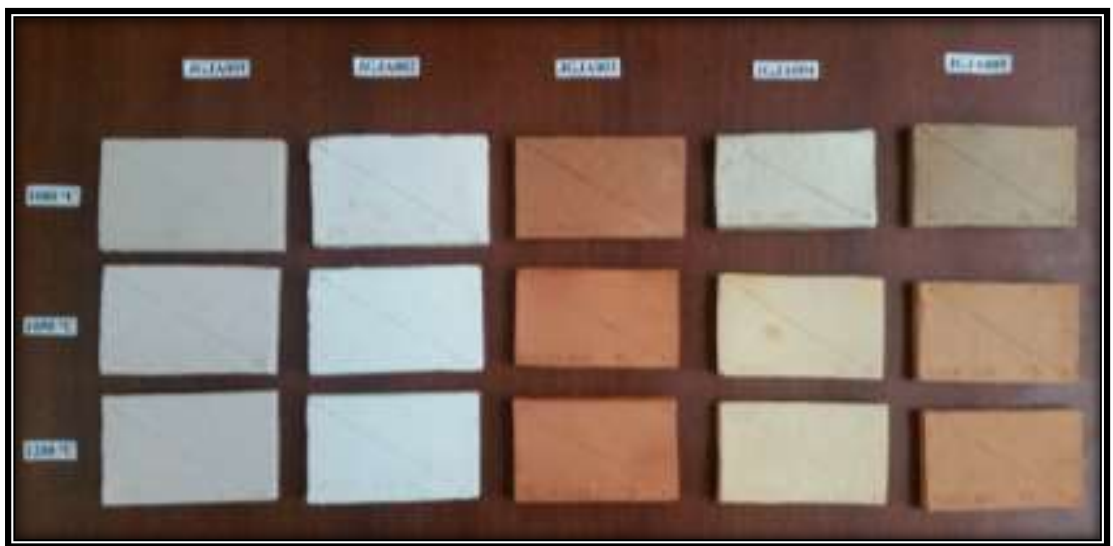

Foto 23: Color de las Placas JGJA $001-J G J A 005$ a temperaturas de $1000^{\circ} \mathrm{C}, 1050{ }^{\circ} \mathrm{C}$ y $1200{ }^{\circ} \mathrm{C}$.

\section{METODOLOGÍA}

Para efectuar este trabajo investigativo en la provincia de Morona Santiago se define un corredor referenciado por el eje vial "troncal amazónica" con sentido norte sur englobando a los cantones Palora, Huamboya, Morona, Sucua, Logroño, Méndez, Tiwinza, Limón Indanza y San Juan Bosco.

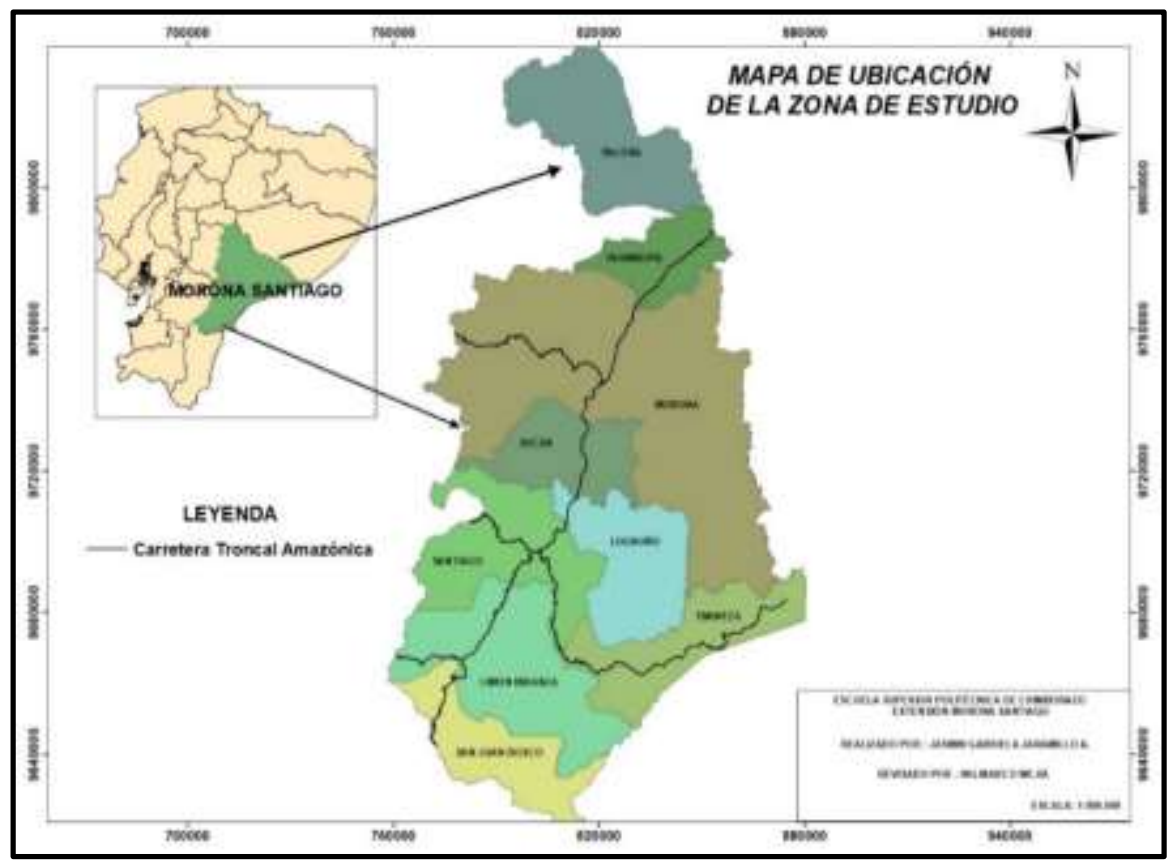

Figura No.1 Mapa de ubicación de la zona de estudio 
Luego del análisis de información bibliográfica de la zona, mapa geológico, carta topográfica, boletines técnicos, indicios determinados en recorridos previos y el conocimiento tradicional proporcionado por los moradores se procede a determinar y ubicar 26 sitios de muestreo (que se detallan en la tabla 1) de materia prima con fin de uso cerámico; dando inicio a la fase de trabajo de campo.

Tabla 1. Coordenadas de las muestras

\begin{tabular}{|c|c|c|}
\hline id_Muestra & $\begin{array}{c}\text { NORTE } \\
(\mathrm{m})\end{array}$ & $\begin{array}{c}\text { ESTE } \\
(\mathrm{m})\end{array}$ \\
\hline JGJA 001 & 9755730 & 815400 \\
\hline JGJA 002 & 9650035 & 772158 \\
\hline JGJA 003 & 9737600 & 815940 \\
\hline JGJA 004 & 9736900 & 816200 \\
\hline JGJA 005 & 9736657 & 816101 \\
\hline JGJA 006 & 9740715 & 817116 \\
\hline JGJA 007 & 9746140 & 819547 \\
\hline JGJA 008 & 9750359 & 814538 \\
\hline JGJA 009 & 9750153 & 814233 \\
\hline JGJA 010 & 9700141 & 793600 \\
\hline JGJA 011 & 9699870 & 793171 \\
\hline JGJA 012 & 9697277 & 798479 \\
\hline JGJA 013 & 9694537 & 799536 \\
\hline JGJA 014 & 9692151 & 795220 \\
\hline JGJA 015 & 9692151 & 795220 \\
\hline JGJA 016 & 9786286 & 184605 \\
\hline JGJA 017 & 9736462 & 815229 \\
\hline JGJA 018 & 9729600 & 813559 \\
\hline JGJA 019 & 9671122 & 783730 \\
\hline JGJA 020 & 9666708 & 779596 \\
\hline JGJA 021 & 9669662 & 781570 \\
\hline JGJA 022 & 9670065 & 782358 \\
\hline JGJA 023 & 9675525 & 793191 \\
\hline JGJA 024 & 9813074 & 829014 \\
\hline JGJA 025 & 9750091 & 816027 \\
\hline JGJA 026 & 9665604 & 826311 \\
\hline
\end{tabular}




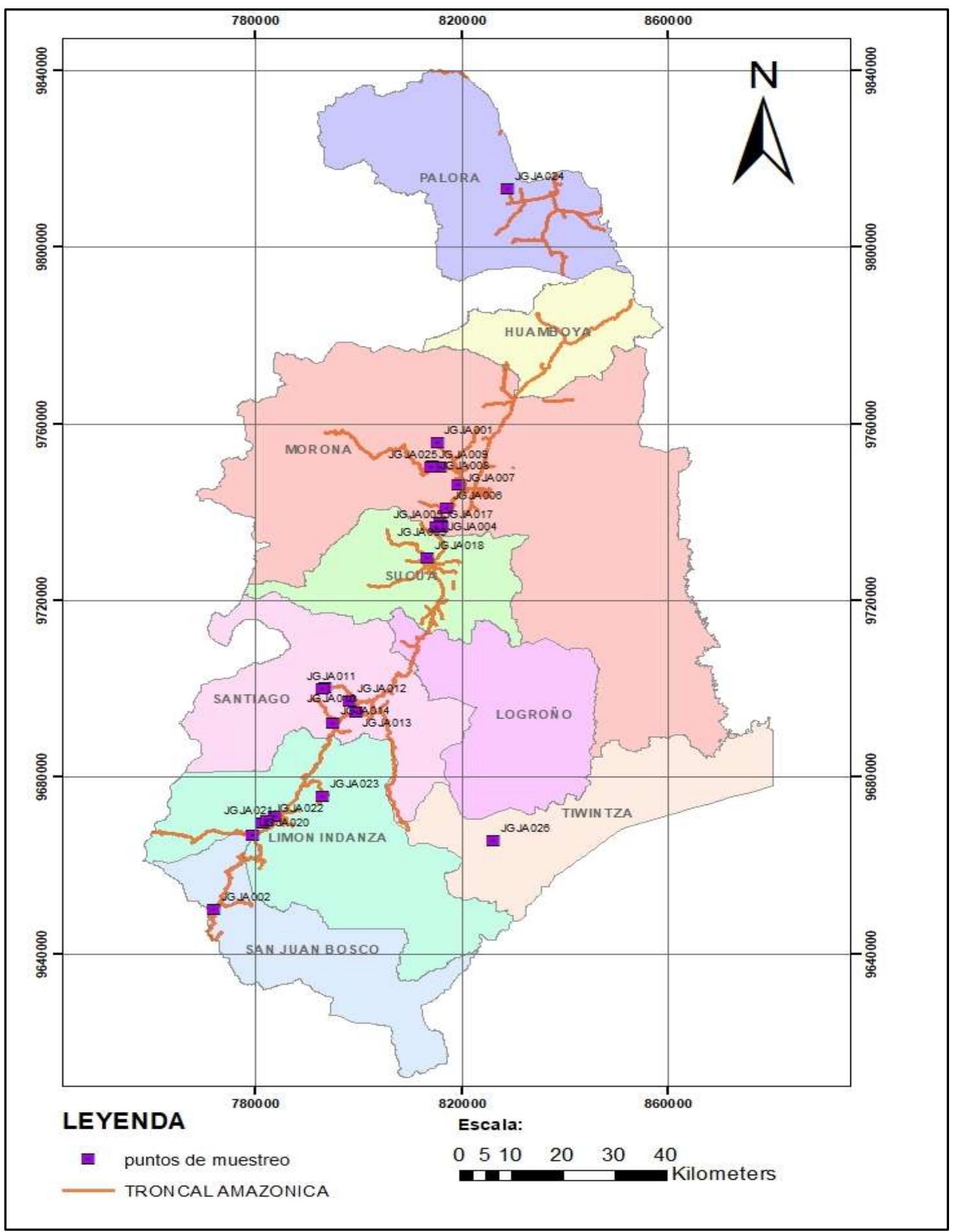

Figura No.2 Mapa con ubicación de las muestras 
Para la toma de muestras sin alterar o modificar las características físico cerámicas del material, se realizó la prospección en lugares donde haya afloramientos expuestos en los ríos, arroyos y en cortes de vías lo que permite acceder a las capas de arcilla o feldespato, que frecuentemente sobresalen. Esta técnica permitió encontrar material de muy buenas características para nuestra investigación; por lo que se procedió a la toma de coordenadas, una breve descripción del afloramiento, toma de muestra representativa y la codificación de cada muestra recolectada. Para llevarla al laboratorio y realizar los ensayos que permitan determinar las propiedades físico-cerámicas de cada una, mediante un análisis del color, plasticidad, refractariedad, porosidad, absorción de agua, contracción a la quema y densidad.

\section{RESULTADOS Y DISCUSIÓN}

TABLAS CON LOS RESULTADOS DE LOS ANÁLISIS FÍSICO-CERÁMICO DE LAS MATERIAS PRIMAS

Tabla 2. Resultados del índice plástico

\begin{tabular}{|c|c|}
\hline MUESTRAS & $\begin{array}{c}\text { ÍNDICE } \\
\text { PLÁSTICO \% }\end{array}$ \\
\hline JEJA 001 & 19,88 \\
\hline JEJA 002 & 20,27 \\
\hline JEJA 003 & 37,10 \\
\hline JEJA 004 & 35 \\
\hline JEJA 005 & 36,10 \\
\hline JEJA 006 & 27,31 \\
\hline JEJA 007 & 15,98 \\
\hline JEJA 008 & 13,54 \\
\hline JEJA 009 & 3,95 \\
\hline JEJA 010 & 14,84 \\
\hline JEJA 011 & 17,71 \\
\hline JEJA 012 & 23,07 \\
\hline JEJA 013 & 18,68 \\
\hline JEJA 014 & 29,79 \\
\hline JEJA 015 & NP \\
\hline JEJA 016 & 10,31 \\
\hline JEJA 017 & 26,23 \\
\hline JEJA 018 & 19,63 \\
\hline JEJA 019 & NP \\
\hline JEJA 020 & 11,68 \\
\hline JEJA 021 & NP \\
\hline JEJA 022 & 10,31 \\
\hline JEJA 023 & 18,69 \\
\hline JEJA 024 & 26,67 \\
\hline JEJA 025 & 14 \\
\hline JEJA 026 & 22,64 \\
\hline & \\
\hline & \\
\hline & \\
\hline
\end{tabular}

Revista Geoespacial 2019 
Tabla 3. Contracción al Secado

\begin{tabular}{|lcccc|}
\hline Muestras & $\begin{array}{c}\text { Longitud en } \\
\text { plástico }\end{array}$ & $\begin{array}{c}\text { Longitud en } \\
\text { seco }\end{array}$ & $\begin{array}{c}\text { Total } \\
(\mathbf{c m})\end{array}$ & $\begin{array}{c}\text { Porcentaje de } \\
\text { contracción } \\
\text { secado }\end{array}$ \\
\hline al
\end{tabular}


Tabla 4. Densidad Real

\begin{tabular}{|c|c|c|c|}
\hline \multicolumn{4}{|c|}{ DENSIDAD REAL $(\mathrm{g} / \mathrm{cm} 3)$} \\
\hline Muestras & $\mathrm{A} 1000^{\circ} \mathrm{C}$ & A $1050^{\circ} \mathrm{C}$ & $1200^{\circ} \mathrm{C}$ \\
\hline JGJA001 & 2,28 & 3,13 & 2,94 \\
\hline JGJA002 & 2,52 & 2,73 & 2,39 \\
\hline JGJA003 & 2,7 & 2,32 & 2,9 \\
\hline JGJA004 & 2,29 & 2 & 2,39 \\
\hline JGJA005 & 2,31 & 2,24 & 2,2 \\
\hline JGJA006 & 2,58 & 2,64 & 2,84 \\
\hline JGJA007 & 2,84 & 2,53 & 2,43 \\
\hline JGJA008 & 2,57 & 2,42 & 2,69 \\
\hline JGJA009 & 2,84 & 2,71 & 3,11 \\
\hline JGJA010 & 2,75 & 2,5 & 2,58 \\
\hline JGJA011 & 2,73 & 2,77 & 2,58 \\
\hline JGJA012 & 2,39 & 2,67 & 2,94 \\
\hline JGJA013 & 2,36 & 2,16 & 2,3 \\
\hline JGJA014 & 2,42 & 2,61 & 2,75 \\
\hline JGJA015 & 2,26 & 2,59 & 2,82 \\
\hline JGJA016 & 2,56 & 2,66 & 2,61 \\
\hline JGJA017 & 2,58 & 2,58 & 2,65 \\
\hline JGJA018 & 2,42 & 2,48 & 2,65 \\
\hline JGJA019 & ........... & ............... & ........... \\
\hline JGJA020 & 2,75 & 2,36 & 2,39 \\
\hline JGJA021 & ........... & ............ & ............ \\
\hline JGJA022 & 2,31 & 2,31 & 2,34 \\
\hline JGJA023 & 2,67 & 2,72 & 2,64 \\
\hline JGJA024 & ........... & 2,54 & 2,53 \\
\hline JGJA025 & 2,65 & 2,58 & 2,73 \\
\hline JGJA026 & 2,84 & 3,44 & 2,74 \\
\hline
\end{tabular}


Tabla 5, Discusión y análisis de resultados

\begin{tabular}{|c|c|}
\hline $\begin{array}{l}\text { Análisis } \\
\text { de } \\
\text { resultados }\end{array}$ & Observación y criterios \\
\hline 1 & $\begin{array}{l}\text { La muestra caolinifera JGJA } 001 \text { se caracteriza por su alta plasticidad, color } \\
\text { blanco y granulometría fina; vitrifica fácilmente en un amplio margen de } \\
\text { temperatura, por ello tiene porcentajes alto de absorción eso significa que es muy } \\
\text { porosa el cual sobrepasa los rangos de } 10-20 \% \text { de absorción de agua establecidos } \\
\text { por Enrique y Amorós en 1985, el rango de contracción es moderado, Se } \\
\text { recomienda realizar la cocción a temperaturas mucho más altas para que llegue a } \\
\text { vitrificarse y pueda servir para porcelanato, para cualquier tipo de cerámica } \\
\text { blanca como loza por su alto contenido de plasticidad y su alta absorción o sino } \\
\text { también se añade este tipo de material para mejorar la blancura de la loza, o las } \\
\text { propiedades de moldeo de los sanitarios, Todo depende para que se vaya a utilizar } \\
\text { ya que las piezas cerámicas de pasta blanca son más apreciadas para los productos } \\
\text { esmaltados, ya que el color blanco permite obtener efectos especiales en el } \\
\text { esmaltado, La contracción recomendada para pasta de porcelana es de } 15 \text { a } 20 \% \\
\text { cocción a } 1350-1400{ }^{\circ} \mathrm{C} \text {, Para pasta de loza se recomienda de } 8 \text { a } 10 \% \text { cocción } \\
\text { de } 1000 \text { - } 1020{ }^{\circ} \mathrm{C} \text {, }\end{array}$ \\
\hline 2 & $\begin{array}{l}\text { La muestra caolinifera JGJA } 002 \text { tiene casi las mismas propiedades que la } \\
\text { muestra JGJA } 001 \text { lo único que varía un poco, son sus valores, por esta razón se } \\
\text { daría el mismo uso ya descrito en la muestra JGJA001, hay que recalcar que su } \\
\text { contenido de absorción es demasiado alto, se recomienda mesclar con algún otro } \\
\text { aditivo para poder mejorar su calidad, }\end{array}$ \\
\hline 3 & $\begin{array}{l}\text { La muestra JGJA 003, tiene una alta plasticidad, su granulometría es muy fina, } \\
\text { debido a su absorción de agua se pude decir que es una muestra porosa, su } \\
\text { contracción es alta lo cual no se podría trabajar por si sola si no tendría que } \\
\text { mezclarse con algún aditivo para mejorar su contracción, Este tipo de pasta } \\
\text { coloreada se aplica para la elaboración cerámica estructural alfarería (ladrillo, } \\
\text { tejas, vasijas, platos, vasos etc,), El rango de cocción que se debe aplicar para la } \\
\text { fabricación de cerámica estructural es de } 900^{\circ} \mathrm{C} \text { a } 1100^{\circ} \mathrm{C} \text {, La contracción } \\
\text { adecuada que tiene que tener es de } 6 \text { a } 8 \% \text { cocción a } 1000^{\circ} \mathrm{C} \text { y } 1020^{\circ} \mathrm{C} \text { para } \\
\text { alfarería, }\end{array}$ \\
\hline 4 & $\begin{array}{l}\text { La muestra JEJA } 004 \text { se caracteriza por tener una alta plasticidad, una } \\
\text { granulometría fina, debido a su porcentaje de absorción se puede decir que esta } \\
\text { muestra es porosa, su color es amarillento tras la cocción, por lo cual se pude } \\
\text { concluir que con las características que presenta se podría aplicar para la } \\
\text { fabricación de azulejos o baldosas cerámicas, El único inconveniente es su } \\
\text { contracción esto hace que sea imposible de utilizarla por sí misma, lo que se } \\
\text { recomienda se use mezclándola a otras arcillas para mejor trabajabilidad, El rango } \\
\text { de \% de agua para la fabricación de azulejos es de (10- } 15 \% \text { entonces cumple } \\
\text { con las especificaciones mencionadas, Esta muestra sufrió deformaciones al igual } \\
\text { de pequeñas fisuras tras la cocción, Es por ello se recomienda una temperatura de } \\
\text { cocción de } 1000{ }^{\circ} \mathrm{C} \text { a } 1020^{\circ} \mathrm{C} \text { con una contracción de } 8 \text { a } 10 \% \text {, }\end{array}$ \\
\hline
\end{tabular}




\begin{tabular}{|c|c|}
\hline 5 & $\begin{array}{l}\text { La muestra JGJA 005, por las características que presentan debido a su color, su } \\
\text { granulometría muy fina, su absorción podemos clasificar que esta muestra a } \\
1200^{\circ} \mathrm{C} \text { no es porosa y llego a vitrificarse, a esta temperatura se presentó una } \\
\text { pequeña deformación al igual que unas pocas fisuras, lo cual se pudo concluir que } \\
\text { esta pasta coloreada puede ser útil para pavimento de gres rojo ,Mientras que a } \\
1000^{\circ} \mathrm{C} \text { hasta } 1050{ }^{\circ} \mathrm{C} \text { la absorción se clasifica como porosa, esta muestra a esa } \\
\text { temperatura sería muy útil para lo que es la cerámica estructural alfarería (ladrillo, } \\
\text { tejas, vasijas, platos, vasos etc,),Su plasticidad es alta, el único inconveniente que } \\
\text { presenta este tipo de materia prima es su excesiva contracción esto hace que sea } \\
\text { imposible de utilizarla por sí misma, lo que se recomendaría que se usen } \\
\text { mezclándolas a otras arcillas para mejor trabajabilidad, La contracción adecuada } \\
\text { que tiene que tener es de } 6 \text { a } 8 \% \text { para la fabricación de cerámica estructural y } \\
\text { alfarería, Para la fabricación de pavimento de gres rojo la contracción adecuada } \\
\text { es de } 10 \text { a } 15 \% \text { cocción } 1250 \text { a } 1280{ }^{\circ} \mathrm{C} \text {, }\end{array}$ \\
\hline 6 & $\begin{array}{l}\text { La muestra JGJA } 006 \text { se caracteriza por tener una alta plasticidad, de } \\
\text { granulometría fina, debido a su porcentaje de absorción podemos que esta } \\
\text { muestra es porosa, su contracción es moderada, Se pudo concluir que esta pasta } \\
\text { coloreada sería muy útil para lo que es la cerámica estructural alfarería (ladrillo, } \\
\text { tejas, vasijas, platos, vasos etc, ) El único inconveniente que se produjo fue una } \\
\text { pequeña deformación ya que el rango temperaturas máximas de cocción para este } \\
\text { tipo de materia prima es de } 1000^{\circ} \mathrm{Ca} 1020^{\circ} \mathrm{C} \text {, La contracción adecuada que tiene } \\
\text { que tener es de } 6 \text { a } 8 \% \text {, }\end{array}$ \\
\hline 7 & $\begin{array}{l}\text { La muestra JGJA 007, por las características que presentan debido a su color, a } \\
\text { su absorción podemos clasificar que esta muestra no es porosa ya que posee una } \\
\text { absorción de agua inferior al } 10 \% \text { a temperatura de } 1050^{\circ} \mathrm{C} \text { y } 1200^{\circ} \mathrm{C} \text {, su } \\
\text { granulometría es muy fina, su contracción total no es muy alta, Lo cual se pudo } \\
\text { concluir que esta pasta coloreada para gres se podría aplicar en pavimentos de } \\
\text { gres rojo, Aunque tenga un factor desfavorable que es la baja plasticidad se podría } \\
\text { compensar con un aditivo de otra arcilla que contenga una alta plasticidad para } \\
\text { tener mejor trabajabilidad, Para la fabricación de pavimento de gres rojo la } \\
\text { contracción adecuada es de } 10 \text { a } 15 \% \text { cocción } 1250^{\circ} \mathrm{C} \text { a } 1280^{\circ} \mathrm{C} \text {, }\end{array}$ \\
\hline 8 & $\begin{array}{l}\text { La muestra JGJA 008, es la idónea para realizar formulación de una pasta para } \\
\text { loza, porque el primer paso para realizar esta formulación es encontrar una arcilla } \\
\text { natural, que cumpla con las siguientes características: de ser de mediana } \\
\text { plasticidad, su contracción total de un } 10 \% \text { a una temperatura de } 1200^{\circ} \mathrm{C} \text {, } \\
\text { absorción de agua de la arcilla cocida de } 6 \% \text { y que no sufra ningún agrietamiento, } \\
\text { Lo cual se pudo concluir con los análisis realizados que esta muestra cumple con } \\
\text { casi todas las especificaciones requeridas para realizar una pasta para loza, lo } \\
\text { único que no se cumplió fue la absorción de agua lo que significa que necesitaría } \\
\text { de algún aditivo para disminuir el porcentaje y llegar con el óptimo, }\end{array}$ \\
\hline 9 & $\begin{array}{l}\text { La muestra JGJA 009, es un feldespato que especialmente por su color y } \\
\text { características que presentan se puede utilizar como aditivo fundente y para el } \\
\text { control de la vitrificación por la acción de los oxidos alcalinos, Este tipo de } \\
\text { feldespato se podría utilizar como un aditivo para loza ya que las especificaciones } \\
\text { son algo menos estrictas en el color, contenido de hierro y mayor presencia de } \\
\text { sodio, }\end{array}$ \\
\hline
\end{tabular}




\begin{tabular}{|c|c|}
\hline 10 & $\begin{array}{l}\text { La muestra JGJA } 010 \text { al igual que la muestra JGJA 008, aun que tienen similares } \\
\text { características se diferencian es su color esto se da debido a las diferentes } \\
\text { proporciones de hierro y cal que tienen las muestras para que se de este tipo de } \\
\text { coloración después de la cocción, de igual manera varían sus valores, pero sigue } \\
\text { siendo idónea para realizar formulación de una pasta para loza, porque el primer } \\
\text { paso para realizar esta formulación es encontrar una arcilla natural, que cumpla } \\
\text { con las siguientes características: de ser de mediana plasticidad, su contracción } \\
\text { total de un } 10 \% \text { a una temperatura de } 1200^{\circ} \mathrm{C} \text {, absorción de agua de la arcilla } \\
\text { cocida de } 6 \% \text { y que no sufra ningún agrietamiento, Lo cual se pudo concluir con } \\
\text { los análisis realizados que esta muestra cumple con casi todas las especificaciones } \\
\text { requeridas para realizar una pasta para loza, lo único que no se cumplió fue la } \\
\text { absorción de agua lo que significa que necesitaría de algún aditivo para disminuir } \\
\text { el porcentaje y llegar con el óptimo, }\end{array}$ \\
\hline 11 & $\begin{array}{l}\text { La muestra JGJA } 011 \text {, tiene una mediana plasticidad, su granulometría es muy } \\
\text { fina, debido a su absorción de agua se pude decir que es una muestra porosa, su } \\
\text { contracción es buena, Este tipo de pasta coloreada se aplica para la elaboración } \\
\text { cerámica estructural alfarería (ladrillo, tejas, vasijas, platos, vasos etc,), Pero a } \\
\text { temperatura de cocción de } 1000^{\circ} \mathrm{C} \text { a } 1020^{\circ} \mathrm{C} \text { con una contracción de } 6 \text { a } 8 \% \text {, Y } \\
\text { también serviría para la elaboración de pasta para loza ya que posee una } \\
\text { contracción dentro de los rangos establecidos de } 8 \text { a } 10 \% \text { el único inconveniente } \\
\text { es su absorción lo que significa que se tendría que agregan algún aditivo para } \\
\text { contrarrestar el valor de esta característica, }\end{array}$ \\
\hline 12 & $\begin{array}{l}\text { La muestra JGJA 012, este tipo de materia prima tiene una buena plasticidad, su } \\
\text { granulometría es fina, el único inconveniente es su absorción y su contracción lo } \\
\text { que se recomienda es modificar a esta materia con algunos aditivos para tener una } \\
\text { buena pasta para el uso de cerámica roja, }\end{array}$ \\
\hline 13 & $\begin{array}{l}\text { La muestra JGJA 013, debido a sus características en especial es su color y su } \\
\text { granulometría este tipo de material solo se utilizaría como aditivo para mejor las } \\
\text { pastas cerámicas ya que su granulometría contiene el } 45 \% \text { de arena y el } 55 \% \text { de } \\
\text { finos, }\end{array}$ \\
\hline 14 & $\begin{array}{l}\text { La muestra JGJA 014, tiene buena plasticidad y excesiva contracción, lo que } \\
\text { significa que presenta las mismas características de una bentonita que cuando } \\
\text { estaba en contacto con el agua se aumentó entre } 10 \text { y } 15 \text { veces su volumen } \\
\text { cuando se secó en su totalidad se redujo demasiado el cual nos indica que es una } \\
\text { bentonita, En conclusión este tipo de material serviría solo como aditivo a la pasta } \\
\text { cerámica para mejorar su plasticidad y su trabajabilidad, pero solo se recomienda } \\
\text { añadirla solo un porcentaje de } 1 \% \text { ya que se pude hacerse pegajosa y difícil de } \\
\text { amasarla, }\end{array}$ \\
\hline 15 & $\begin{array}{l}\text { La muestra JGJA } 015 \text {, es un feldespato que especialmente por su color y } \\
\text { características que presentan se puede utilizar como aditivo fundente y para el } \\
\text { control de la vitrificación por la acción de los oxidos alcalinos, este tipo de } \\
\text { feldespato se podría utilizar como un aditivo para las porcelanas ya que la } \\
\text { principal característica que debe ser un color de cocción blanco o un color crema, } \\
\text { mínimo contenido de hierro, alto contenido de óxidos alcalinos, }\end{array}$ \\
\hline
\end{tabular}




\begin{tabular}{|c|c|}
\hline 16 & $\begin{array}{l}\text { La muestra JGJA } 016 \text { al igual que la muestra JGJA017 se caracteriza por tener } \\
\text { una mediana plasticidad, una granulometría fina, debido a su porcentaje de } \\
\text { absorción se puede decir que esta muestra es porosa, su color es amarillento tras } \\
\text { la cocción, la única diferencia es su contracción baja, lo cual se pude concluir que } \\
\text { con las características que presenta; se podría aplicar para la fabricación de } \\
\text { azulejos o baldosas cerámicas, También se podría aplicarse para la formulación } \\
\text { de una pasta para Loza el único inconveniente seria su absorción debido a que } \\
\text { debe cumplir con las siguientes características: de ser de mediana plasticidad, su } \\
\text { contracción total de un } 10 \% \text {, absorción de agua de la arcilla cocida de } 6 \% \text { y que } \\
\text { no sufra ningún agrietamiento, Se recomienda mezclarla con algunos aditivos } \\
\text { para mejoras las características de esta muestra en el caso de la absorción, }\end{array}$ \\
\hline 17 & $\begin{array}{l}\text { La muestra JGJA } 017 \text { se caracteriza por tener una mediana plasticidad, una } \\
\text { granulometría fina, debido a su porcentaje de absorción se puede decir que esta } \\
\text { muestra es porosa, su color es amarillento tras la cocción, lo cual se pude concluir } \\
\text { que con las características que presenta se podría aplicar para la fabricación de } \\
\text { azulejos o baldosas cerámicas, También se podría aplicarse para la formulación } \\
\text { de una pasta para Loza el único inconveniente seria su contracción y su absorción } \\
\text { debido a que debe cumplir con las siguientes características: de ser de mediana } \\
\text { plasticidad, su contracción total de un } 8 \text { a } 10 \% \text {, absorción de agua de la arcilla } \\
\text { cocida de } 6 \% \text { y que no sufra ningún agrietamiento, Se recomienda mezclarla con } \\
\text { algunos aditivos para mejoras las características de esta muestra, En el caso de } \\
\text { para hacer baldosas o azulejos el único inconveniente es la contracción, }\end{array}$ \\
\hline 18 & $\begin{array}{l}\text { La muestra JGJA } 018 \text { se caracteriza por tener una alta plasticidad, una } \\
\text { granulometría fina, un buen color; debido a su porcentaje de absorción a } 1000^{\circ} \mathrm{C} \\
\text { se puede decir que no es porosa, su contracción es óptima porque está dentro de } \\
\text { los rangos de } 10 \text { a } 15 \% \text { establecidos para realizar pastas de gres, Partir de las } \\
\text { temperaturas de } 1050^{\circ} \mathrm{C} \text { a } 1200^{\circ} \mathrm{C} \text { el porcentaje de absorción es el óptimo para } \\
\text { fabricación de azulejos o baldosas cerámicas, al igual que su contracción, }\end{array}$ \\
\hline 19 & $\begin{array}{l}\text { Nota,- no se pudo realizar las pruebas de contracción después de la cocción, ni } \\
\text { las de la absorción, ya que al trayecto de ponerlas en el horno se trisaron, se } \\
\text { desasieron debido a su nula plasticidad y compactación, }\end{array}$ \\
\hline 20 & $\begin{array}{l}\text { La muestra JGJA } 020 \text { se caracteriza por tener una mediana plasticidad, una } \\
\text { granulometría fina, su color, Esta muestra se utilizaría para pastas rojas en si para } \\
\text { cerámica estructural y alfarería a una temperatura de } 1000^{\circ} \mathrm{C} \text { ya que a esa } \\
\text { temperatura cumple los rangos establecidos, el único inconveniente seria su } \\
\text { absorción, También se podría utilizarlas a temperatura de } 1050^{\circ} \mathrm{C} \text { solo tendría } \\
\text { que bajar el } 1 \% \text { de su contracción par que se ideal para alfarería o cerámica } \\
\text { estructural, Si se desea aplicar esta muestra para pasta para loza se recomienda } \\
\text { que la absorción de la muestra baja aun porcentaje de } 6 \% \text { ya que tiene la } \\
\text { contracción y plasticidad óptima para este tipo de uso, }\end{array}$ \\
\hline 21 & $\begin{array}{l}\text { Nota,- No se pudo realizar las pruebas de contracción después de la cocción, ni } \\
\text { las de la absorción, ya que al trayecto de ponerlas de ponerlas en el horno se } \\
\text { trisaron, se desasieron debido a su nula plasticidad y compactación, }\end{array}$ \\
\hline 22 & $\begin{array}{l}\text { La muestra caolinifera JGJA 022, su característica es un material de medina } \\
\text { plasticidad, de color blanco tras la cocción, presenta moderada contracción a la } \\
\text { cocción, Este tipo de arcilla se puede considerar como caolines refractarios, se lo } \\
\text { utilizaría para ladrillos y piezas usadas en la construcción de hornos industriales } \\
\text { o a su vez en cerámica blanca se recomienda mejorar su absorción de agua, }\end{array}$ \\
\hline
\end{tabular}




\begin{tabular}{|c|c|}
\hline 23 & $\begin{array}{l}\text { La muestra JGJA 023, la única característica favorable de este tipo de materia } \\
\text { prima es su alta plasticidad debido a su granulometría fina, ya que su contracción } \\
\text { y absorción son desfavorables, así mismo se presentó pequeñas fisuras y } \\
\text { deformidades tras la cocción, Lo cual se pudo concluir que esta muestra solo } \\
\text { serviría como aditivo para mejora la plasticidad de las pastas cerámicas, }\end{array}$ \\
\hline 24 & $\begin{array}{l}\text { La muestra JGJA } 024 \text { este tipo de material se caracterizan por presentar una alta } \\
\text { plasticidad y color blanco tras cocción, es de grano finísimo, sufre un } \\
\text { encogimiento de } 11 \% \text {, en el proceso tras la cocción a } 1200^{\circ} \mathrm{C} \text {, Es un producto } \\
\text { poroso se la clasifico de esta manera porque está dentro del rango establecido por } \\
\text { Enrique y Amorós en } 1985 \text {, entre } 10-20 \% \text { de absorción de agua, Esto nos lleva a } \\
\text { concluir que este material es apto para la fabricación de pavimentos, ladrillería de } \\
\text { alta calidad, loza, porcelana sanitaria y materiales refractarios, También sirve } \\
\text { para mezclar con otros tipos de arcillas que carecen de plasticidad, }\end{array}$ \\
\hline 25 & $\begin{array}{l}\text { La muestra JGJA } 025 \text {, tiene mediana plasticidad, su granulometría es fina, se } \\
\text { podría utilizar para cerámica estructural y alfarería ya que tiene una buena } \\
\text { contracción a } 1000^{\circ} \mathrm{C} \text {, está dentro de los rangos establecidos lo único } \\
\text { desfavorable es su absorción de agua ya que sobrepasa los valores óptimos para } \\
\text { la fabricación de este tipo de cerámica, }\end{array}$ \\
\hline 26 & $\begin{array}{l}\text { La muestra JGJA 026, tiene mediana plasticidad, su granulometría muy fina, se } \\
\text { podría utilizar para cerámica estructural y alfarería ya que tiene una buena } \\
\text { contracción, el único inconveniente es su desfavorable absorción se recomienda } \\
\text { mezclar con otro tipo de arcilla para mejorar su calidad y bajar su absorción, }\end{array}$ \\
\hline
\end{tabular}

\section{CONCLUSIONES}

A partir de las pruebas físico-cerámicas realizadas a las muestras recolectadas, se pudo evidenciar que existe en la provincia de Morona Santiago yacimientos no metálicos aprovechables que perfilan de buenas características para el uso cerámico bien sea para cerámica blanca o cerámica roja, Del corredor referencial en este estudio se desprende que en los cantones Palora, Huamboya Morona, Sucua, Méndez, Tiwinza, Limón Indanza y San Juan Bosco, existen materia prima aprovechable para cerámica, mientras que en el cantón Logroño no se encontró materiales de buenas características,

De los 26 materiales estudiados, las muestras JGJA 003, JGJA 005, JGJA 006, JGJA 007, JGJA 010, JGJA 011, JGJA 012, JGJA 013, JGJA 014, JGJA 018, JGJA 020, JGJA 023, JGJA 025, JGJA 026 son para cerámica roja, aptos para materiales de construcción (ladrillos, tejas, etc,) y productos de alfarería, La muestra JGJA 024 presenta características muy favorables para la fabricación de pavimentos, ladrillería de alta calidad, loza, porcelana sanitaria y materiales refractarios,

Las muestras JGJA 001, JGJA 002, JGJA 008, JGJA022 son para cerámica blanca, Las muestras JGJA 004, JGJA 017 y JGJA 016 son aptas para baldosas o lozas, Las muestras JGJA 009 y JGJA 015 son feldespatos que pueden ser útiles como aditivo fundente para la cerámica,

La mayor parte de las muestras recolectas de la zona estudiada está constituida por material idóneo para el uso de alfarería, 


\section{REFERENCIAS}

Báez, N, Núñez, R,, Loachamín, R, y Tirado, R, (2005), Rocas y Minerales Industriales en el Ecuador, Quito,

Cargua, L, y Encalada, D, (1997), Investigación De Materiales Cerámicos Alivianados Y Sus Posibles Aplicaciones, Cuenca,

Caruso, N, (1986), Cerámica Viva, Omega, S,A, Barcelona

Daly, E, (1998), MANUAL DEL CERAMISTA, Ediciones Daly S,L, Málaga

Díaz, L, y Torrecillas, R, (2002), Arcillas Cerámicas: una revisión de sus distintos tipos, significados y aplicaciones, Boletín De La Sociedad Española De Cerámica y Vidriado, 460 ,

Dolors, R, I, (2005), CERÁMICA ARTÍSTICA, Parramón Ediciones, S,A, Barcelona,

Fierro, F, (1987), La Minería de la Jurisdicción del C,R,E,A, (Azuay, Cañar Y Morona Santiago), Cuenca,

Grim, R, (1953), Clay Mineralogy, McGraw-Hill Book Company,

Hoffstetter y Bristow, R, (1977), Léxico Estratigráfico Internacional, Centre National de la Recherche Scientifique, Paris,

J,F, B, (1997), El Caolín: composición, estructura, génesis y aplicaciones, Cerámica y Vidrio, 7 , 121

Jami, A, y Zuñiga, F, (1997), Porcelana Fosfática con el uso de Materia Prima Nacional, Cuenca,

Mejía, N, (2011), Arcilla, Facultad de Ingenieria y Arquitectura. Universidad Privada ALAS PERUANAS. Huaraz, Peru.

Moreno, M, y Polo, M, (2012), Análisis y Elaboración de un Ladrillo de Barro Producido con los Materiales del Cantón Chambo que Cumplan con las Especificaciones de Calidad Necesarias, Riobamba,

PLAN NACIONAL DE DESARROLLO DEL SECTOR MINERO, (2016), MINISTERIO DE MINERÍA, Ecuador

Rhodes, D, (1990), Arcilla y Vidriado para el Ceramista, EDICIONES CEAC, S,A, Barcelona, España. 